\title{
Investigation of flame propagation in a partially premixed jet by high- speed-Stereo-PIV and acetone-PLIF
}

\author{
J. Weinkauff ${ }^{1}$, P. Trunk ${ }^{1}$, J.H. Frank ${ }^{2}$, M.J. Dunn ${ }^{3}$, A. Dreizler ${ }^{1}$, B. Böhm ${ }^{4}$
}

1: Fachgebiet Reaktive Strömungen und Messtechnik, Technische Universität Darmstadt Jovanka-Bontschits-Straße 2, D - 64287 Darmstadt, Germany

2: Combustion Research Facility, Sandia National Laboratories, Livermore, CA 94551-0969, USA

3: School of Aerospace, Mechanical and Mechatronic Engineering, The University of Sydney, NSW 2006, Australia

4: Fachgebiet Energie- und Kraftwerkstechnik, Technische Universität Darmstadt Jovanka-Bontschits-Straße 2, D - 64287 Darmstadt, Germany

Corresponding author: Benjamin Böhm, Jovanka-Bontschits-Straße 2, D - 64287 Darmstadt, Germany, Fax: +49 6151 166555, e-mail: bboehm@ekt.tu-darmstadt.de

\section{Colloquium \\ Diagnostics}




\begin{abstract}
This paper presents an experimental study of flame propagation through a partially-premixed flow following ignition. A combination of simultaneous high-speed acetone planar laser induced fluorescence (PLIF) and stereoscopic particle image velocimetry (SPIV) was utilized for time-resolved measurements of mixture fraction, flow field and flame position. This provides access to the major quantities needed to characterize non-premixed flames. High quality mixture fraction measurements with signal-to-noise ratios up to 120 for unity mixture fraction were made feasible using a combination of a conventional high-speed laser at $10 \mathrm{kHz}$ for LIF excitation and a wavelet based de-noising algorithm to reject camera noise. It was observed that flame propagation in the far-field of a partially-premixed jet takes place in a premixed mode, with the flame propagating through highly stratified mixtures until it approaches locations containing mixtures outside the flammability limits. In these areas the flame recedes and further propagation is controlled by mixing processes of air and fuel. Even though flame propagation is then mixing-controlled, the flame is not observed to switch into a non-premixed mode. Instead, mixing ahead of the flame takes place until locally premixed flammable mixtures are recovered for subsequent flame propagation.
\end{abstract}

Keywords

High-speed measurements, Acetone PLIF, stereo PIV, flame propagation, mixture fraction 


\section{Introduction}

Ignition and propagation of turbulent non- and partially-premixed flames is of crucial importance for a variety of combustion applications, such as altitude-relight of aviation gasturbines, combustion in Diesel engines and flame spreading over liquid or solid fuels. Success or failure of flame initiation and subsequent flame propagation is influenced by local properties of the turbulent flow and mixing field. Detailed experimental investigations in canonical benchmark systems such as turbulent jets can improve our physical understanding and contribute to the development of combustion models.

Mixtures in the far-field of non-premixed jets are highly stratified with alternating streaks of very lean and rich mixtures [1]. When the jet flow is ignited, flames propagate into the stratified mixture in a premixed mode as long as the reactant mixture remains within the flammability limits [2]. The stratified premixed flame may transition into a mixing controlled scenario where air-fuel mixtures are too rich or lean to burn. For such a case, there are several possible mechanisms for the flame to proceed:

a) Flame propagation comes to rest or the flame extinguishes until subsequent upstream mixing provides flammable mixtures to continue propagation or allow for reignition.

b) Flames switch into a non-premixed mode where fuel and air are provided from the respective sides of the flame.

c) Tribrachial flames form combining properties of premixed and non-premixed flames.

For case a), the flame remains a premixed flame even though its propagation speed might be controlled by mixing upstream of the flame rather than flame wrinkling. For case c), an edge flame may develop, depending on the local mixture fraction $(\xi)$ gradient [2]. In laminar flows, tribrachial flames may form in regions of low $\xi$ gradients with a leading triple-point, accompanied by lean and rich premixed flame branches and a trailing non-premixed flame 
[3]. For steeper $\xi$ gradients, the tribrachial structure collapses into edge flames, while for very low mixture gradients no clear edge may be observable [2]. In turbulent flows, the turbulenceflame interactions may lead to further ambiguities in the detection of an edge flame.

Flame propagation in turbulent non-premixed or partially-premixed mixtures has been studied in lifted turbulent jet flames [4]. During upstream propagation, large-scale flow structures affect the flame structure and can lead to localized extinction [5]. High-speed PIV measurements have shown little correlation between the propagation speeds of lifted flames and the turbulence intensity [6]. Propagation of spark-ignited turbulent non-premixed edge flames in a turbulent partially-premixed counter-flow has been investigated previously [7]. In that study, the hypothesis that failed ignition events resulted from variations of fuel-air ratios could not be verified because time-resolved $\xi$ fields were not measured.

Multidimensional high-speed $\xi$ and velocity measurements are required to better understand the mechanisms of flame propagation in turbulent stratified flows. The determination of $\xi$ either requires simultaneous measurements of all major-species and temperature or the measurement of conserved scalars. Planar mixture fraction measurements were performed using Raman/Rayleigh [8], difference Rayleigh-scattering [9] and tracer-LIF [10-13]. TracerLIF using organic substances such as acetone or 3-pentanone is applicable only prior to combustion where decomposition reactions have not yet occurred. Gordon et al. [13] demonstrated the feasibility of time-resolved planar measurements in an isothermal jet using acetone-PLIF at $9.5 \mathrm{kHz}$. Depending on acetone seeding densities and the imaging system they achieved signal-to-noise ratios (SNR) up to $\sim 200$ for $\xi=1$ at the nozzle exit.

Instantaneous $\xi$ and scalar dissipation rates are influenced by local properties of the turbulence field, such as strain or vorticity. Therefore, combined velocity/scalar measurements are imperative. Simultaneous Raman/Rayleigh/Laser-Doppler-velocimetry approaches were shown to be feasible at single points [14]. Simultaneous acetone-PLIF and PIV measurements at $10 \mathrm{~Hz}$ have provided information on $\xi$ and two velocity components 
[11]. Recently, this technique was extended to $10 \mathrm{kHz}$ in a feasibility study in an isothermal jet [12].

In the present study, propagation of a partially-premixed flame following ignition on the jet's centreline is studied. The focus is on an experimental analysis of the above described scenario a), in which flame propagation may stagnate and become controlled by mixing. Multiparameter high-speed diagnostics are used to simultaneously measure temporal dynamics of flame-front propagation, $\xi$ fields in the unburned region upstream the flame-front and three components of the velocity fields. This phenomenon can be captured in a relatively modest Reynolds number jet flow, which enables measurements that are highly temporally resolved with respect to the flow time-scales. In addition, it reduces complications in interpreting highspeed planar imaging measurements of a three-dimensional flow field.

\section{Experimental setup}

\section{Turbulent jet burner}

A sketch of the experimental setup is shown in Figure 1. Experiments were performed in a jet burner consisting of a vertically aligned $350 \mathrm{~mm}$ long tube with an inner diameter of $6 \mathrm{~mm}$, sufficiently long for fully developed pipe flow at the exit. The nozzle exit was tapered to a sharp edge to minimize recirculation and was surrounded by a co-flow with an inner diameter of $130 \mathrm{~mm}$ providing air with an exit velocity of $0.2 \mathrm{~m} / \mathrm{s}$. The Reynolds number of the jet flow was $\sim 2500$ based on the inner tube diameter. Fuel issuing from the jet was a blend of methane, acetone, nitrogen and oxygen. The volumetric ratio of methane, nitrogen and oxygen was 0.5/0.2/0.3 and controlled by electronic mass-flow meters (Bronkhorst). Saturated acetone vapour was seeded into the gas mixture using two sequential bubblers containing liquid acetone that was maintained at a temperature of $20^{\circ} \mathrm{C}$. The temperature of the acetoneseeded gas mixture was monitored by a thermocouple at the exit of the bubblers. The volumetric acetone seeding fraction was $25 \%$. The gases in the jet were mixed $1 \mathrm{~m}$ upstream 
of the nozzle exit. Oxygen was added to the fuel stream to increase the stoichiometric mixture fraction $\xi_{\text {st }}$ to 0.132 from 0.072 for the pure methane/acetone mixture. This measure increased the acetone-LIF signal at stoichiometric conditions. Nitrogen was added primarily to lower adiabatic flame temperatures. For SPIV-measurements, the co-flow was seeded with $\mathrm{MgO}$ particles $(\sim 1 \mu \mathrm{m})$. The jet flow was ignited by focusing a pulsed Nd:YAG laser $(\lambda=532 \mathrm{~nm})$ at the jet centreline $200 \mathrm{~mm}$ downstream of the nozzle exit. After data acquisition the flame was blown off and the experiment was repeated every $20 \mathrm{~s}$. Three hundred individual ignition events were recorded. A laminar reference-jet $4 \mathrm{~mm}$ in diameter was placed into the laser path $150 \mathrm{~mm}$ ahead of the probe volume, and the resulting acetone-LIF signal was recorded on a separate high-speed CMOS camera. The reference-jet flow was obtained by diverting $5 \%$ of the fuel stream from the main jet, and thus the composition of the two jets was identical.

\section{High-speed-Stereo-PIV and acetone-PLIF setup}

The fourth-harmonic of a diode-pumped dual-head Nd:YAG laser (Edgewave CX16II-E) operated at $10 \mathrm{kHz}$ was used for acetone-PLIF mixture fraction measurements. The laser was operated in a burst mode of 400 pulses per sequence resulting in $\sim 1.5 \mathrm{~mJ}$ per pulse. A PellinBroca prism was used to separate UV light at $266 \mathrm{~nm}$. For SPIV-measurements, a frequencydoubled Nd: $\mathrm{YVO}_{4}$ dual-cavity laser (Edgewave IS4II-DE, $4.0 \mathrm{~mJ}$ ) was operated at $10 \mathrm{kHz}$. The UV and $532 \mathrm{~nm}$ beams for simultaneous PLIF and SPIV measurements were combined using a high-reflectivity $266 \mathrm{~nm}$, high-transmissivity $532 \mathrm{~nm}$ dichroic beam-splitter. The laser beams were formed into light sheets with a thickness of $250 \mu \mathrm{m}$. For SPIV, pulses from the two laser heads were separated by $50 \mu \mathrm{s}$. The PLIF laser was fired in-between the SPIV laser pulses.

The acetone fluorescence emission was imaged onto a high-speed CMOS camera (HSS6) operated at $10 \mathrm{kHz}$ and synchronized to the UV laser. For high collection efficiency, a combination of a Canon $85 \mathrm{~mm} \mathrm{f} / 1.2$ lens and a $160 \mathrm{~mm}$ achromatic lens was used which 
resulted in a projected pixel dimension of $38 \times 38 \mu \mathrm{m}^{2}$. A second high-speed CMOS camera (HSS5) equipped with an $85 \mathrm{~mm}$ Zeiss f/1.4 lens recorded the LIF-signal from the referencejet. The SPIV setup was similar to the one presented in [15] using a pair of CMOS cameras (HSS6) operated at $20 \mathrm{kHz}$ and equipped with Sigma $180 \mathrm{~mm}$ f/2.8 macro lenses. The cameras were oriented in forward scattering orientation on either side of the laser sheet with an offset-angle of $30^{\circ}$. Scheimpflug adaptors were used to correct for off-axis defocussing. The field-of-view of the system was $13 \times 13 \mathrm{~mm}^{2}$.

\section{Data processing}

\section{Acetone-PLIF for mixture fraction ( $\xi$ ) imaging}

To take into account effects of non-uniform CMOS-chip sensitivity, individual pixel-offset, and non-linearity, we calibrated the CMOS camera using an Ulbricht Sphere [16]. Each PLIF image was corrected for the pixel-response before background subtraction. To improve SNR, we applied a wavelet adaptive thresholding (WATR) technique for de-noising [17]. A previously developed 1D wavelet de-noising algorithm [17] was extended to two dimensions, with the primary modifications being the use of bi-orthogonal 2D waveletfunctions [18] and a 2D stationary wavelet transform. The CMOS camera-noise data necessary for the de-noising algorithm to statistically characterize the noise processes were determined using dark and Ulbricht Sphere measurements. Using a 3-sigma confidence interval in wavelet transform space and applying the thresholding algorithm, $99.7 \%$ of the camera-noise is rejected while preserving the optical imaging resolution.

Synthetic images of isotropic turbulence obtained by the inflow-generator presented in [19] together with typical detector characteristics and length-scales from the experiment were used to validate the WATR performance. Figure 2 shows a synthetic dissipation-spectrum together with a spectrum superimposed with the characteristic camera-noise. The comparison of the 
WATR method with a state-of-the art adaptive Wiener-filter highlights its superior performance for measurements where the camera-noise characteristics are known.

After de-noising, a flat-field image correction was performed followed by image de-warping to transfer pixel coordinates into physical space coordinates. The resulting images were then corrected for attenuation of the laser beam by acetone absorption by applying the LambertBeer law in an iterative routine that computed the local acetone concentration. Beam-steering was insignificant and therefore neglected. The validity of cross-sections used in the absorption correction was confirmed by tracking acetone concentration profiles in spatially homogeneous regions of the laminar reference-jet. Vertical fluorescence profiles obtained from the reference-jet were used to account for laser profile and shot-to-shot intensity variations. In addition the reference-jet was used to quantify unity $\xi$. The laser energy flux was almost an order of magnitude lower than in the work of Bryant et al. [20] where laser fluxes of $350 \mathrm{~mJ} / \mathrm{cm}^{2}$ still resulted in linear LIF signals.

An uncertainty analysis was performed by measuring the SNR of the acetone-LIF signal near the nozzle exit, where no secondary air was yet entrained and $\xi$ is by definition unity. The precision was calculated as the standard deviation from all pixels within a 50x100 pixel area of 400 individual snapshots. For the de-noised images the precision was $0.8 \%$ corresponding to a SNR of 120 for $\xi=1$ (SNR of raw images was 60 ). The SNR decreased to $\sim 15$ at stoichiometric conditions.

\section{Temperature dependence of acetone-LIF signal}

The determination of $\xi$ from the acetone-LIF signal is straightforward for isothermal conditions in the mixing field upstream of the flame, whereas in the close vicinity of the flame-front with rising temperatures the interpretation of the acetone-LIF signal is complicated by local variations of total number densities, varying acetone absorption cross- 
sections and quantum-yields, as well as decomposition reactions. Simultaneous measurements of temperature would have resolved some of these ambiguities but were not available in the present study. As a result, we excluded the analysis of acetone-PLIF signals in the region directly adjacent to the flame. To select an appropriate exclusion distance, we performed a calculation of a freely propagating premixed laminar flame in a stoichiometric mixture of the jet and co-flow streams. The calculation was performed using the PREMIX module of Chemkin [21] in connection with the San Diego chemical mechanism [22] and an acetone sub-mechanism [23]. Figure 3 shows calculated profiles of temperature (T), relative acetone number density $\left(\mathrm{ND}_{\text {Acetone }}\right)$ and relative LIF signal along the flame normal direction. The flame-normal distance is given by $\mathrm{x}_{\mathrm{f}}$ with the flame-front, defined by the steepest temperature gradient, at the coordinate's origin. Relative acetone number density and LIF signal were normalized by their corresponding maximum values in the non-reacted mixture. AcetonePLIF signals were calculated by the model proposed by Thurber et al. [24] using local acetone number densities, temperatures, and the combined effects of the temperature-dependent absorption cross-section and fluorescence quantum-yield. Deviation of the normalized LIF signal from unity marks the region close to the flame-front (grey shaded region in Figure 3) where local fluctuations in the acetone-LIF signal cannot be attributed solely to variations of $\xi$. To avoid such a bias and constrain the error to less than $5 \%$ in our analysis, we excluded the area $0.6 \mathrm{~mm}$ ahead of the flame-front from further analysis.

\section{SPIV and flame-front detection}

Spatial calibration, de-warping of the SPIV images and matching to the acetone-PLIF images were accomplished by using a three-dimensional reference target (Type7, LaVision). Image cross-correlation and vector validation was performed with a multi-pass iterative crosscorrelation algorithm (Davis 8, LaVision). Final interrogation window size and overlap were 
32 pixels and $75 \%$, respectively. This corresponds to a vector spatial resolution of $0.94 \mathrm{~mm}$ and spacing of $0.23 \mathrm{~mm}$.

The Mie-scattering images from the SPIV system were also used to identify the flame-front from the particle density jump across the flame. Therefore an image processing procedure iteratively applied Gaussian filters with decreasing kernel sizes (50x50 pixels down to $15 \times 15$ pixels). A large kernel size was applied first to estimate the flame-front location. Subsequently, the raw image was filtered using the $15 \times 15$ pixel kernel in the region of the estimated flame-front and the 50x50 pixel kernel in the remainder of the image. Large kernel sizes increased the robustness to seeding inhomogeneities while smaller kernels maximized the accuracy of the procedure in the vicinity of the flame. To identify the particle density jump a threshold was defined from the signal distribution of the filtered image as the local minimum between low signals in the hot flow and high signals in the cold flow.

\section{Results and discussion}

\section{Isothermal jet}

Figure 4 shows an individual snapshot of the isothermal flow. The SPIV vector field (every 30th vector shown) is superimposed on the mixture fraction $(\xi)$ field (top). The finger-like mixing structures are typical of large-scale vortex roll-up within the shear layer that transports ambient unmixed air deep into the jet [1]. For this snapshot, this appears as an extended streak of ambient air inclined to the centreline direction. These streaks are bounded by regions of high scalar dissipation rate $\left(\chi=D(\nabla \xi)^{2}\right.$ with the diffusion coefficient $\left.D=1\right)$ in accordance with findings of [11]. This is evident from Figure 4 (middle) where scalar dissipation rates are shown with directions of the in-plane $2 \mathrm{D}$ minimum principal strain $\vec{e}_{2 D \min }$ superposed. Principal strain is represented by the in-plane components of the strain tensor transformed to its principal form assuming a $2 \mathrm{D}$ flow within the plane. Due to $3 \mathrm{D}$ effects, the value of $\sigma_{2 D \min }(2 \mathrm{D}$ minimum principal strain) is not necessarily negative [11]. In this work, we use 
$\sigma_{2 D m i n}$, usually compressive strain, especially to identify regions where the flow decelerates. Dissipation structures appear very smooth and demonstrate the effectiveness of the wavelet de-noising. The dissipation structures are relatively thin and elongated which is in agreement with a sheet-like topology in 3D space. Similar to the observations in Ref. [11], regions of high dissipation correlate with regions of compressive strain (bottom), and the compressive strain is aligned predominantly normal to the dissipation structures.

To provide confidence in the accuracy of $\xi$ measurements, we compare the centerline profile of the mean mixture fractions $\bar{\xi}$ to known scaling laws for isothermal jets. Figure 5 shows $\bar{\xi}$ along the centreline ranging from $x / D=1$ to 20 . The scaling-law is given by $\bar{\xi}=5.4(x)$ $\left.\left(D\left(\frac{\rho_{\text {jet }}}{\rho_{\text {coflow }}}\right)^{0.5}\right)\right)$ with the jet diameter D and densities $\rho$ of jet and co-flow [25]. The scalinglaw holds true for $\mathrm{x} / D>7$. Up to $\mathrm{x} / D=2, \bar{\xi}$ is unity within the core region of the jet. Subsequently, $\bar{\xi}$ decreases due to turbulent mixing with ambient air. For $\mathrm{x} / D>7$, measurements are in fair agreement with the scaling-law, despite the low Reynolds number which is slightly above transition, providing confidence in the $\xi$ measurements.

\section{Propagating flame}

Figure 6 shows a temporal sequence of a flame as it propagates, from top to bottom, through the partially-premixed $\xi$ field. The region of interest is located $120-130 \mathrm{~mm}$ downstream of the jet's exit. The flame-front (deduced from the particle density jump) is represented by red lines. As stated before, the region $0.6 \mathrm{~mm}$ ahead of these lines is excluded from the analysis. The solid and dashed black contours indicate $\xi_{\mathrm{st}}$ and the lean flammability limit, respectively, for a freely propagating unstrained flame. In the $1^{\text {st }}$ column, the $\xi$ field is overlaid with the vectors of the in-plane velocity components. The out-of-plane velocity for this sequence (not shown) is within $+/-0.5 \mathrm{~m} / \mathrm{s}$ for the field-of-view (FOV). The $2^{\text {nd }}$ column quantifies the 
corresponding magnitude of $\sigma_{2 D \min }$. The sequence highlights the transient nature of the experiment, with the flame propagating through the FOV. The $10 \mathrm{kHz}$ repetition rate of the measurement system results in well-resolved time-series measurements of the flame, the local $\xi$ and flow field and their interaction. Although the FOV spans only $10 \times 15 \mathrm{~mm}^{2}$, several tens to a hundred measurement frames can be acquired during flame transit.

The selected example shows typical finger-like structures of $\xi$ alternating between lean and rich mixtures. While pure air is found on the left bottom of the FOV, other regions have lean mixtures that are outside of the flammability limits. These lean regions form barriers that inhibit flame propagation. Figure 7 shows the pdf of $\xi$ extracted from flame-parallel lines 1 $\mathrm{mm}$ ahead of the flame for all frames from 0 to $8 \mathrm{~ms}$. The flammability limits (vertical lines) highlight the regime where the flame could propagate in a premixed mode. The actual flammability limits may be narrower because of fluid dynamic straining. The mean $\xi$ at this downstream location is slightly below the stoichiometric $\xi$. The bimodal distribution extends beyond the flammability limits and reflects the presence of finger-like structures. It has a peak at very lean mixtures that are below the lean flammability limit and another slightly to the rich side of $\xi_{\text {st. }}$

In the first frames (0-1 ms) of Figure 6, the flame propagates approximately opposite to the flow direction. Corresponding $\xi$ fields are within the flammability limits. This indicates that the flame burns in a premixed mode. High values of compressive strain are generated in front of the flame due to the opposite directions of flow and upstream flame propagation. On top of the flame-front, peak values of extensive strain are observed that are related to heat-release and the accompanied flow acceleration. At $1.5 \mathrm{~ms}$, the flame slows down as it reaches the entrained pocket of air (diagonally oriented blue streak). At this instant, compressive strain structures in the direct vicinity of the flame vanish and the extensive strain at the flame-front decreases significantly. Subsequently (3-6 ms), the flame reverses its direction of propagation and is convected downstream (frames in-between not shown). Upstream flame propagation is 
inhibited due to too lean mixtures in front of the flame. In the present sequence, approximately $4 \mathrm{~ms}$ are needed for the fuel to diffuse from the rich structures into the lean region ahead of the previous flame-front to again provide flammable mixtures for re-ignition and to re-establish flame propagation. Starting from $6 \mathrm{~ms}$, the flame continues its propagation in the upstream direction. High extensive strain at the flame-front is recovered again and is accompanied by high compressive strain ahead of the flame-front.

For a more quantitative assessment of the mixing field ahead of the flame-front, Figure 8 shows instantaneous profiles of $\xi$, extracted perpendicular to the flame-front at $0 \mathrm{~ms}$ along the dashed grey line marked in Figure 6. The position of the line remains fixed for the rest of the sequence. The profiles are presented in flame coordinates with the flame-front positioned at $\mathrm{x}_{\mathrm{f}}=0 \mathrm{~mm}$. During the initial $2.0 \mathrm{~ms}$ (Figure $8 \mathrm{top}$ ), the profiles correspond to the flame propagation into the region of entrained air that appears as the afore-mentioned finger-like structure. At $0 \mathrm{~ms}$, entrainment of air has decreased $\xi$ ahead of the flame $\left(\mathrm{x}_{\mathrm{f}}=3.3 \mathrm{~mm}\right)$ to less than 0.04. Between the flame and this minimum a local peak $(\xi=0.13)$ is observed. Within the following $1 \mathrm{~ms}$, fuel contained in this peak-area is continuously consumed by the upstream propagating flame until $\xi=0.06$ is reached. In these lean mixtures, the combustion process is retarded and flame propagation finally stagnates. At this stage local extinction might have occurred. This hypothesis cannot be proven by our measurements because the density jump provides no information on the instantaneous heat release rate but only marks the border between burned (hot) and unburned (cold) regions. In the vicinity of the flame, $\xi$ continues to decrease even though fuel rich mixtures are observed further upstream. This region of low $\xi$ could be excess air originating from previous combustion of lean regions that mix with adjacent unburned gases. The very lean mixture in combination with the onset of a backward moving "flame-front" is indicative of local flame extinction. The $\xi$ gradient broadens over the next $4 \mathrm{~ms}$ while the flame recedes. Fuel diffuses into the yet un-burnable lean mixtures. $\xi$ increases until flammability limits are recovered and flame propagation is re-initiated. Figure 
8 (bottom) shows profiles of $\xi$ for $6-7.5 \mathrm{~ms}$, when the flame continues to burn in the upstream direction. As the flame moves, the profiles are no longer aligned perfectly perpendicular to the flame. Although this misalignment is small, it may contribute to variations in the $\xi$ gradient, along with any 3D-effects. Nevertheless, the increase in $\xi$ gradients due to increasing compressive strain ahead of the flame is significant.

\section{Conclusions}

This paper presents an experimental study of flame propagation through a partially-premixed flow following ignition. The diagnostics and data processing is presented together with first results. A combination of simultaneous time-resolved measurements of mixture fraction $(\xi)$, flow field and flame position provides access to the major quantities needed to characterize non-premixed flames. Acetone-PLIF was used for $\xi$ measurements. The application of a wavelet adaptive thresholding method provided high quality $\xi$ measurements with an SNR up to 120 at $\xi=1$ using a conventional high-speed laser at $10 \mathrm{kHz}$ for LIF excitation.

The results suggest that in the far-field of partially- or non-premixed jet flows, flame propagation proceeds in a premixed mode. A premixed flame burns through highly stratified mixtures until it approaches locations containing mixtures outside the flammability limits. These areas of alternating very lean and rich mixtures outside the flammability limits are caused by turbulent mixing and appear as finger-like structures. At these instants, flames recede or even extinguish. Further flame propagation is controlled by mixing processes of air and fuel. Even though flame propagation is then mixing-controlled, the flame is not observed to switch into a non-premixed mode. Instead, mixing ahead of the flame takes place until locally premixed flammable mixtures are recovered for subsequent flame propagation. A large database of 300 individual ignition events is available and will be analysed to investigate the leading edge on a statistical base to see if it preferentially propagates through stoichiometric mixtures. 


\section{Acknowledgments}

Financial support by Deutsche Forschungsgemeinschaft (EXC 259) is gratefully acknowledged. J.H. Frank was supported by the U.S. Department of Energy, Office of Basic

Energy Sciences, Division of Chemical Sciences, Geosciences, and Biosciences. Sandia National Laboratories is a multiprogram laboratory operated by Sandia Corporation, a Lockheed Martin Company, for the U.S. Department of Energy under contract DE-AC04-94AL85000. 


\section{References}

[1] W.J.A. Dahm, P.E. Dimotakis, AIAA Journal 25 (1987), 1216-1223.

[2] E. Mastorakos, Prog. Energy Combust. Sci. (2009) 35, 57-97.

[3] J. Buckmaster, Prog. Energy Combust. Sci. (2002) 28, 435-475.

[4] K.M. Lyons, Prog. Energy Combust. Sci. (2007) 33, 211-231.

[5] I. Boxx, C. Heeger, R. Gordon, B. Böhm, M. Aigner, A. Dreizler, W. Meier, Proc. Combust. Inst. 32 (2009), 905-912.

[6] R.L. Gordon, I. Boxx, C. Carter, A. Dreizler, W. Meier, Flow Turb.Combust.(2012) 88, 503-527.

[7] C. Heeger, B. Böhm, S. Ahmed, R. Gordon, I. Boxx, W. Meier, A. Dreizler, E. Mastorakos, Proc. Combust. Inst. 32 (2009), 2957-2964.

[8] J.H. Frank, K.M. Lyons, D.F. Marran, M.B. Long, S.H. Starner, R.W. Bilger, Proc. Combust. Inst. 25 (1994), 1159-1166.

[9] J. Fielding, J.H. Frank, S.A. Kaiser, M.D. Smooke, M.B. Long, Proc. Combust. Inst. 29 (2003), 2703-2709.

[10] A.G. Hsu, V. Narayanaswamy, N.T. Clemens, J.H. Frank, Proc. Combust. Inst. 33 (2011), 759-766.

[11] J. Rehm, N.T. Clemens, (1999) AIAA-99-0676.

[12] J.D. Miller, J.B. Michael, M.N. Slipchenko, S. Roy, T.R. Meyer, J.R. Gord, Appl. Phys. B 113 (2013), 93-97.

[13] R.L. Gordon, C. Heeger, A. Dreizler, Appl. Phys. B 96 (2009), 745-748.

[14] R.W. Dibble, V. Hartmann, R.W. Schefer, W. Kollmann, Exp. Fluids 5 (1987), 103113.

[15] P.J. Trunk et al., Proc. Combust. Inst. 34 (2012), 3565-3572.

[16] V. Weber, J. Brübach, R.L. Gordon, A. Dreizler, Appl. Phys. B 103 (2011), 421-433.

[17] M.S. Sweeney, S. Hochgreb, M.J. Dunn, R.S. Barlow, Combust. Flame 160 (2013), 322-334.

[18] A. Cohen, I. Daubechies, J.-C. Feauveau, Comm. Pure Appl. Math. 45 (1992) 485-560.

[19] M. Klein, A. Sadiki, J. Janicka, J. Comp. Physics 186 (2003) 652-665.

[20] R.A.Bryant, J.M.Dobar, J.F.Driscoll, Exp. Fluids 28/5 (2000), 471-476.

[21] R.J. Kee, F.M. Rupley, J.A. Miller, et al., CHEMKIN-PRO Release 15113, Reaction Design Inc., San Diego (2012).

[22] Chemical-Kinetic Mechanisms for Combustion Applications, San Diego Mechanism web page, Mechanical and Aerospace Engineering (Combustion Research), University of California at San Diego, 2005, available at http://combustion.ucsd.edu.

[23] S. Pichon, G. Black, N. Chaumeix, M. Yahyaoui, J. Simmie, H. Curran, R. Donohue, Combust. Flame 156 (2009), 494-504.

[24] M.C. Thurber, F. Grisch, B.J. Kirby, M. Votsmeier, R.K. Hanson, Appl. Opt. 37 (1998), 4963. 
[25] K.M. Tacina, W.J.A. Dahm, J. Fluid Mech. (2000) 415, 23-44. 


\section{List of Figure Captions}

Figure 1: Experimental setup.

Figure 2: Generic test-case for the validation of wavelet de-noising method: Synthetic spectrum, synthetic spectrum with superimposed noise, noisy spectrum after Wiener filtering and wavelet de-noising.

Figure 3: Normalized acetone number densities $N D_{\text {acetone }}$ and temperature based on laminar flame calculations and normalized LIF signals deduced from the Thurber model as a function of flame distance $\mathrm{x}_{\mathrm{f}}$. The region where the LIF signal is influenced by the flame is grey shaded.

Figure 4: Snapshot of the isothermal flow: Flow field (every 30th vector shown) superposed on the $\xi$ field (top), direction of minimum principal strain $\vec{e}_{2 D \min }$ superposed on scalar dissipation field (middle) and minimum principal strain $\sigma_{2 D \min }$ (bottom); the origin of the coordinate system is on the centerline at the nozzles exit.

Figure 5: Axial profile of mean mixture fraction along the centerline together with the slope determined by the scaling-law for isothermal jets.

Figure 6: Individual temporal sequence of flame (red line) propagation: $1^{\text {st }}$ and $3^{\text {rd }}$ column shows the mixture fraction field with superposed velocity vectors (every $30^{\text {th }}$ vector shown) together with $\xi_{\text {st }}=0.132$ (black line) and lower flammability limit (black dashed line). Note that $\xi$ is biased in a $0.6 \mathrm{~mm}$ region ahead of the flame. $2^{\text {nd }}$ and $4^{\text {th }}$ column gives the magnitude of minimum principal strain $\sigma_{2 D m i n}$. Along the dashed gray line in the first frame $\xi$ profiles were extracted shown in Figure 8; the origin of the coordinate system is on the centerline at the nozzles exit.

Figure 7: Pdf of $\xi$ extracted from lines $1 \mathrm{~mm}$ ahead of the flame for the complete image sequence. Flammability limits are indicated by vertical dashed lines.

Figure 8: Profiles of $\xi$ extracted along the grey dashed line (see Figure 6). The axial coordinate $\mathrm{x}_{\mathrm{f}}$ gives the distance to the flame-front. 


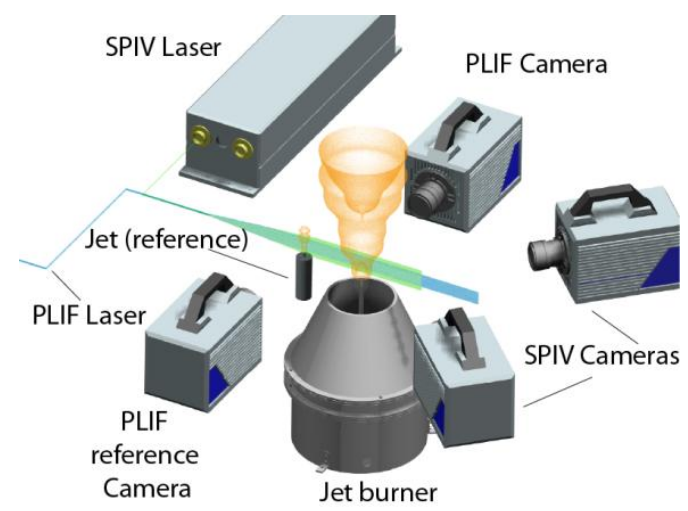

Figure 1: Experimental setup.

Single-column width (bw print) 


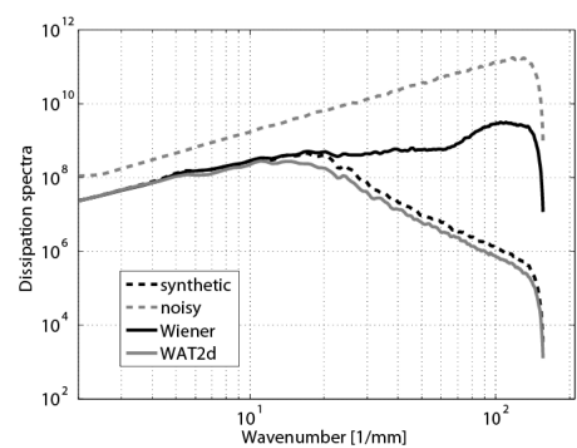

Figure 2: Generic test-case for the validation of wavelet de-noising method: Synthetic spectrum, synthetic spectrum with superimposed noise, noisy spectrum after Wiener filtering and wavelet de-noising.

Single-column width (bw print) 


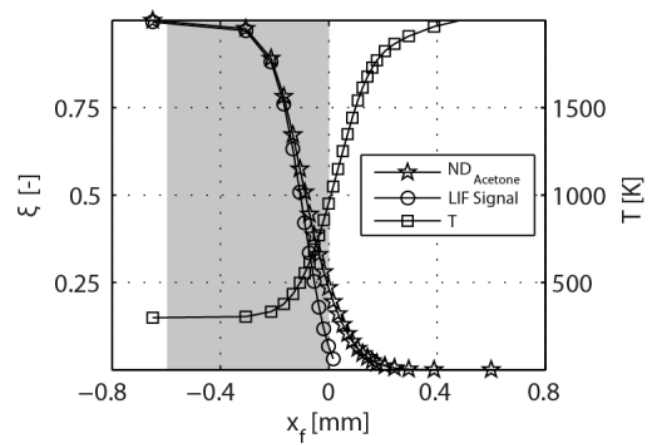

Figure 3: Normalized acetone number densities $N D_{\text {acetone }}$ and temperature based on laminar flame calculations and normalized LIF signals deduced from the Thurber model as a function of flame distance $\mathrm{x}_{\mathrm{f}}$. The region where the LIF signal is influenced by the flame is grey shaded.

Single-column width (bw print) 


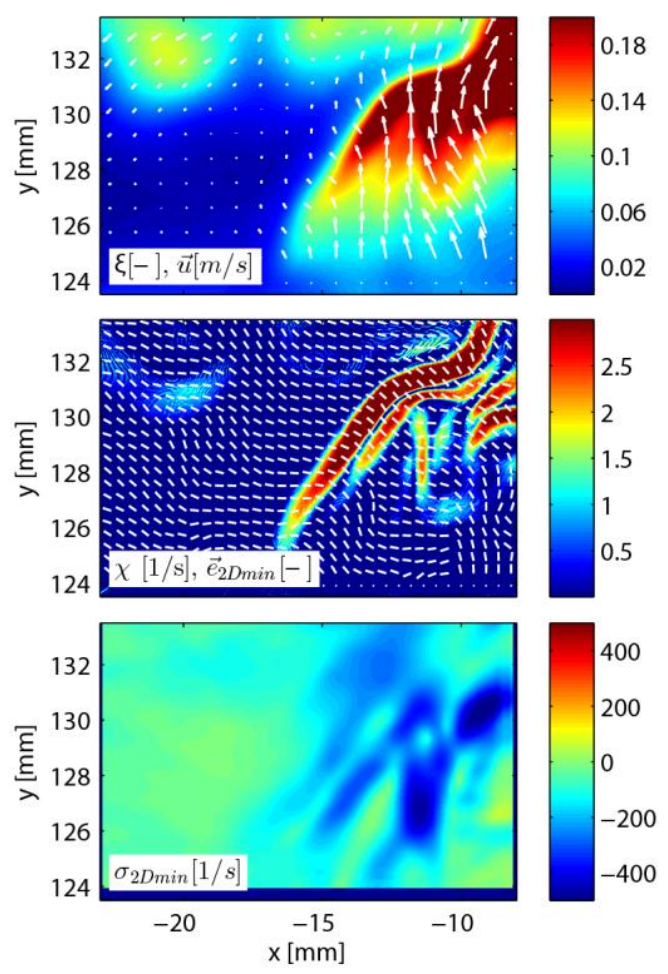

Figure 4: Snapshot of the isothermal flow: Flow field (every 30th vector shown) superposed on the $\xi$ field (top), direction of minimum principal strain $\vec{e}_{2 D \min }$ superposed on scalar dissipation field (middle) and minimum principal strain $\sigma_{2 D \min }$ (bottom); the origin of the coordinate system is on the centerline at the nozzles exit.

Single-column width (color print) 


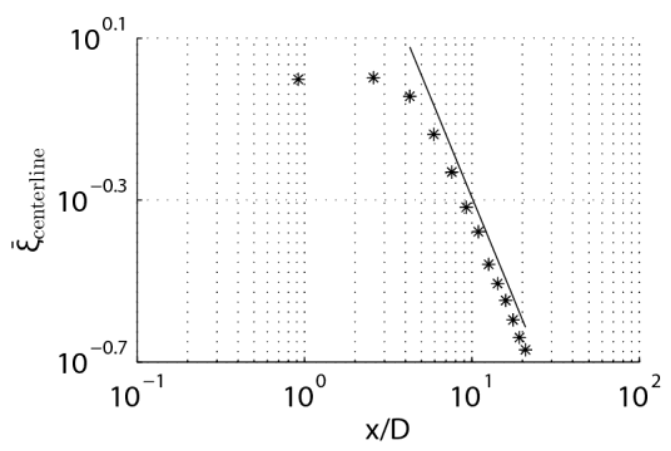

Figure 5: Axial profile of mean mixture fraction along the centerline together with the slope determined by the scaling-law for isothermal jets.

Single-column width (bw print) 

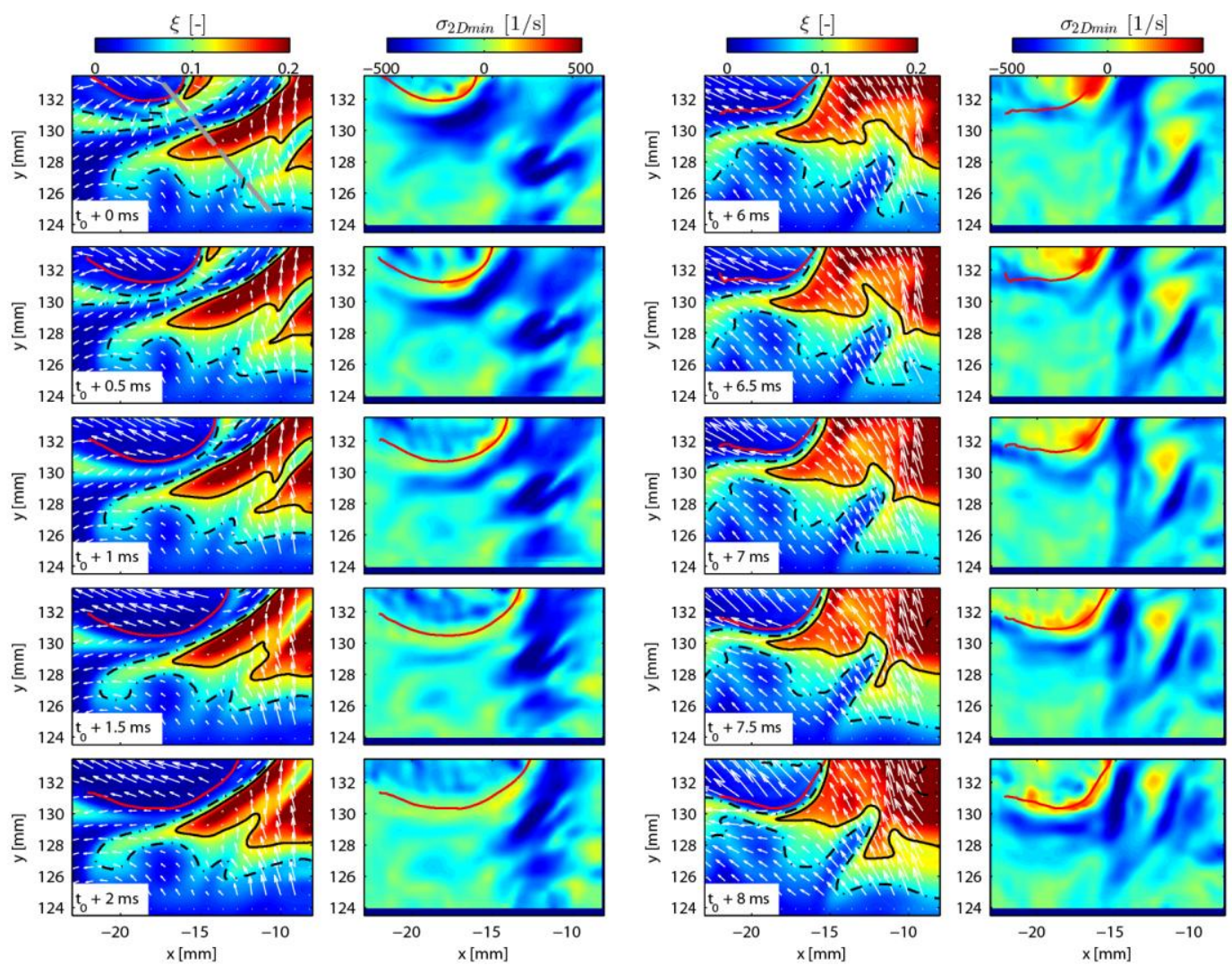

Figure 6: Individual temporal sequence of flame (red line) propagation: $1^{\text {st }}$ and $3^{\text {rd }}$ column shows the mixture fraction field with superposed velocity vectors (every $30^{\text {th }}$ vector shown) together with $\xi_{\text {st }}=0.132$ (black line) and lower flammability limit (black dashed line). Note that $\xi$ is biased in a $0.6 \mathrm{~mm}$ region ahead of the flame. $2^{\text {nd }}$ and $4^{\text {th }}$ column gives the magnitude of minimum principal strain $\sigma_{2 D m i n}$. Along the dashed gray line in the first frame $\xi$ profiles were extracted shown in Figure 8; the origin of the coordinate system is on the centerline at the nozzles exit.

Double-column width (color print) 


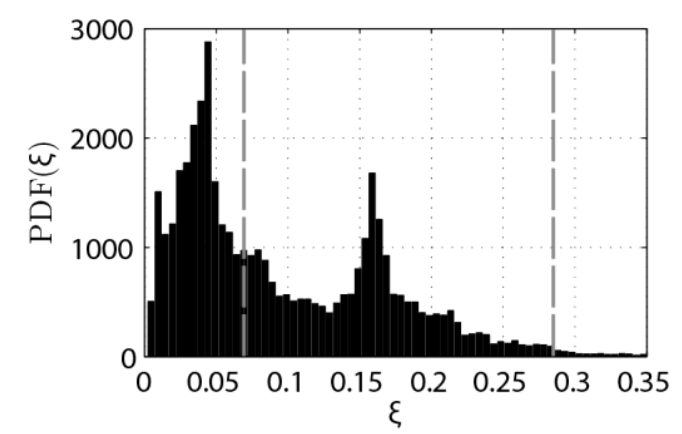

Figure 7: Pdf of $\xi$ extracted from lines $1 \mathrm{~mm}$ ahead of the flame for the complete image sequence. Flammability limits are indicated by vertical dashed lines.

Single-column width (bw print) 


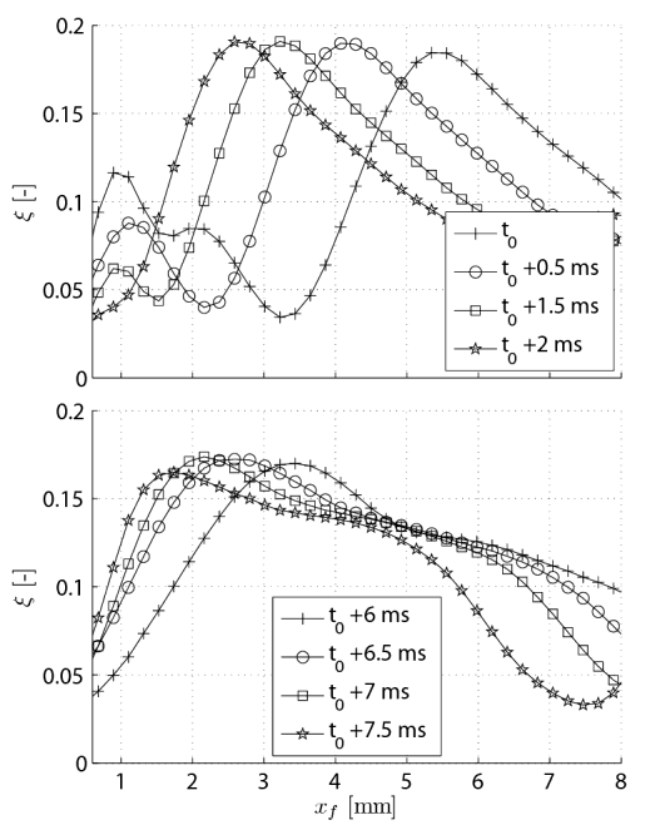

Figure 8: Profiles of $\xi$ extracted along the grey dashed line (see Figure 6). The axial coordinate $\mathrm{x}_{\mathrm{f}}$ gives the distance to the flame-front.

Single-column width (bw print) 